\title{
Fractals, nonextensive statistics, and QCD
}

\author{
Airton Deppman $\odot,{ }^{1,2, *}$ Eugenio Megías $\odot,{ }^{2}$ and Debora P. Menezes $\odot^{3}$ \\ ${ }^{1}$ Instituto de Física, Rua do Matão 1371-Butantã, São Paulo-SP, CEP 05508-090 Brazil \\ ${ }^{2}$ Departamento de Física Atómica, Molecular y Nuclear and Instituto Carlos I de Física \\ Teórica y Computacional, Universidad de Granada, E-18071 Granada, Spain \\ ${ }^{3}$ Depto de Física, CFM-Universidade Federal de Santa Catarina Florianópolis, \\ SC-CP. 476-CEP 88.040-900, Brazil
}

(Received 15 January 2020; accepted 24 January 2020; published 19 February 2020; corrected 21 April 2020)

\begin{abstract}
In this work, we analyze how scaling properties of Yang-Mills field theory manifest as self-similarity of truncated n-point functions by scale evolution. The presence of such structures, which actually behave as fractals, allows for recurrent nonperturbative calculation of any vertex. Some general properties are indeed independent of the perturbative order, what simplifies the nonperturbative calculations. We show that for sufficiently high perturbative orders a statistical approach can be used, the nonextensive statistics is obtained, and the Tsallis index, $q$, is deduced in terms of the field theory parameters. The results are applied to QCD in the one-loop approximation, where $q$ can be calculated, resulting in a good agreement with the value obtained experimentally. We discuss how this approach allows us to understand some intriguing experimental findings in high energy collisions, as the behavior of multiplicity against collision energy, long-tail distributions, and the fractal dimension observed in intermittency analysis.
\end{abstract}

DOI: 10.1103/PhysRevD.101.034019

\section{INTRODUCTION}

Yang-Mills field (YMF) theory is a prototype theory to describe three among the four known natural interactions, namely, strong, weak, and electromagnetic, with only gravitational force left aside. ${ }^{1}$ The importance of such category of field theory is summarized in the Standard Model, which is a single framework to describe all three interactions in a unified formalism. Renormalization group invariance is a fundamental aspect of the Yang-Mills field theory, playing an important role in the renormalization of the theory after the ultraviolet divergences are subtracted [2-4]. The theory was extremely successful in calculations for QED and weak interactions, providing accurate and precise descriptions for the observed phenomena. QCD has proved to be more challenging, since the calculation methods applied to the other two forces are not appropriate to provide accurate results for strong interacting systems. In this scenario, QCD has been tested in high energy elementary particle interactions, where asymptotic freedom allows

\footnotetext{
*deppman@if.usp.br

${ }^{1}$ Recently, a formulation based on Yang-Mills theory has been proposed for gravity [1].

Published by the American Physical Society under the terms of the Creative Commons Attribution 4.0 International license. Further distribution of this work must maintain attribution to the author(s) and the published article's title, journal citation, and DOI. Funded by SCOAP ${ }^{3}$.
}

for the application of perturbative methods, and by lattice QCD (LQCD), which is a numerical approach based on QCD first principles.

Fractals are complex systems with internal structure presenting scale invariance and self-similarity. Fractal measures, contrary to more conventional quantities for which an increase in resolution results in the same measured value with increased precision, yield different values for different resolutions. A classical example is the length of coastlines [5]. Since its proposal about three decades ago, the concept of fractals has found many applications in Mathematics, Arts, Biology and Complex Systems in general. A nice introduction to the subject and its applications can be found in Refs. [5,6] and more formal descriptions in Ref. [7]. Among the most important features of fractals are the scaling properties, where the internal structure of the fractal is equal to the main fractal but with a reduced scale, self-symmetry, and fractional dimensions.

Tsallis statistics generalizes Boltzmann-Gibbs one by introducing a nonadditive form for entropy, what leads to nonextensivity of some quantities. The effects of Tsallis statistics have been explored since it was proposed, in the late 80s [8,9], but its full meaning and fundamentals are still far from being completely understood. The $q$-deformed entropy functional that underlies nonextensive statistics depends on a real parameter, $q$, that determines the degree of nonadditivity of the functional, and in the limit $q \rightarrow 1$, it becomes additive and the standard Boltzmann-Gibbs entropy is recovered. 
Our goal is to show a subtle link between renormalization group invariance of YMF, fractals, and the Tsallis nonextensive statistics. For that aim, we analyze how the scaling properties of a Yang-Mills field theory lead to recurrence relations that allow nonperturbative calculations, which amount to a self-similar behavior of truncated n-point diagrams by scale evolution. Essentially, these amplitudes are shown to behave as fractals by evolving the scale to the ultraviolet region and the calculation of vertices even in high perturbative orders becomes possible due to a simple recurrence formula. For sufficiently high perturbative order, the nonextensive statistics are obtained, and the Tsallis index, $q$, is deduced in terms of the field parameters.

Our work is organized as follows: we first introduce the basic concepts of the Yang Mills theory and how a system of interacting partons relates to fractals. Then a connection between the partonic self-similarity and the nonextensive statistics is established. In other words, we will demonstrate that renormalizable field theories lead to fractal structures, which can be studied, from a thermodynamical point of view, with Tsallis statistics. Finally, as an application, wellknown QCD scaling properties are used to calculate, for the first time, the $q$ value in terms of QCD fundamental parameters. Some consequences of our results are discussed, in particular the fractal dimension, which is experimentally observed by intermittency analysis, and the behavior of particle multiplicity as a function of the collision energy, which is here related to the nonextensivity and to the fractal dimension of the hot and dense system formed at high energy collisions. This is an intriguing aspect of experimental data which can be explained in a simple way from QCD by the present approach, as well as other properties of high energy collisions, such as multiplicity dependence on the collision energy, long-tail distributions, and fractal dimension observed in intermittency studies.

\section{FORMALISM}

The simplest scale free non-Abelian gauge field theory has Lagrangian density including bosons and fermions given by

$$
\mathcal{L}=-\frac{1}{4} F_{\mu \nu}^{a} F^{a \mu \nu}+i \bar{\psi}_{j} \gamma_{\mu} D_{i j}^{\mu} \psi_{j}
$$

where

$$
F_{\mu \nu}^{a}=\partial_{\mu} A_{\nu}^{a}-\partial_{\nu} A_{\mu}^{a}+g f^{a b c} A_{\mu}^{b} A_{\nu}^{c}
$$

and

$$
D_{i j}^{\mu}=\partial_{\mu} \delta_{i j}-i g A^{a \mu} T_{i j}^{a},
$$

where $\psi$ and $A$ represent, respectively, the fermion and the vector fields, with $f^{a b c}$ being the structure constants of the group and $T^{a}$ the matrices of the group generators in the fermion representation.

The UV regularized vertex functions are related to the renormalized vertex functions with renormalized parameters, $\bar{m}$ and $\bar{g}$, as

$$
\Gamma(p, m, g)=\lambda^{-D} \Gamma(p, \bar{m}, \bar{g}) .
$$

This property is mathematically described by the renormalization group equation, which introduce the beta function, which allowed to show that QCD is asymptotically free [10-12]. Such equation is known as CallanSymanzik equation [13-15] and is given by

$$
\left[M \frac{\partial}{\partial M}+\beta_{\bar{g}} \frac{\partial}{\partial \bar{g}}+\gamma\right] \Gamma=0,
$$

where $M$ is the scale parameter, and the $\beta$ function is defined as

$$
\beta_{\bar{g}}=M \frac{\partial \bar{g}}{\partial M} .
$$

$D=D_{o}+d$, with $D_{o}$ being the natural dimension of the phase space. In general, $d$ is not an integer; therefore, the scaling dimension, $D$, may be fractionary. The parameter $\gamma$ is the anomalous dimension given by a combination of the scaling dimensions of the fields $\psi$ and $A$.

In the irreducible one-particle representation, self-energy is taken into account by using the effective parton mass, $\bar{m}$, what allows a reduction of complexity in the calculation, since only proper vertices must be considered. As schematically shown in Fig. 1, the renormalization group invariance means that, after proper scaling, the loop in a higher order graph is identical to a loop in lower orders [10-12]. The renormalization group invariance of the vertex function is a direct consequence of the Callan-Symanzik equation, and it is of fundamental importance in what follows. When effective charges and masses are used, the line of the respective field in Feynman diagram represent an effective particle or state, since parts of the diagram

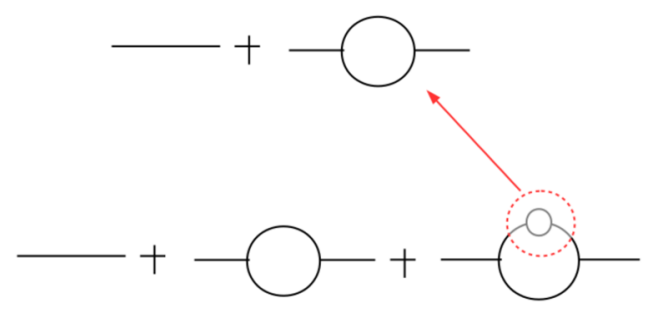

FIG. 1. Diagrams showing the scaling properties of Yang-Mills fields: a loop in higher order is identical, after proper scaling, to a diagram in lower order. 
representing self-energy contribution are omitted because they are already taken into account by the renormalization. Such diagrams are called irreducible graphs. We will next refer to the physical system represented by irreducible graphs as effective parton. In irreducible graphs, the only allowed vertices, called proper vertices, are related to the creation of an effective parton.

Despite the great reduction in complexity when irreducible graphs are used, still complex graphs are unavoidable in performing perturbative calculations, especially for QCD, since vertex functions may include several orders in perturbative approximation. We will show that some additional simplification may be achieved under some circumstances that are relevant in hadron structure and in multiparticle production. Preliminary results of the approach developed here were discussed in Ref. [16]. From now on, we refer to proper vertices as interactions.

\section{A. Statistical description of the partonic state}

The time evolution of an initial partonic state is

$$
|\Psi\rangle \equiv|\Psi(t)\rangle=e^{-i H t}\left|\Psi_{o}\right\rangle
$$

The state $\Psi$ can be written as

$$
|\Psi\rangle=\sum_{n}\left\langle\Psi_{n} \mid \Psi\right\rangle\left|\Psi_{n}\right\rangle
$$

with the basis states $\left|\Psi_{n}\right\rangle$ corresponding to a fixed number, $n$, of interactions in the vertex function. In the perturbative method, each proper vertex gives rise to a term in the Dyson series, and at any time, $t$, the partonic state is given by

$$
|\Psi\rangle=\sum_{\{n\}}(-i)^{n} \int d t_{1} \ldots d t_{n} e^{-i H_{o}\left(t_{n}-t_{n-1}\right)} g \ldots e^{-i H_{o}\left(t_{1}-t_{o}\right)}\left|\Psi_{o}\right\rangle,
$$

where $g$ represents the interaction and $t_{n}>t_{n-1}>\ldots$ $>t_{1}>t_{o}$, and $\sum_{\{n\}}$ runs over all possible terms with $n$ interaction vertices. Observe that $\left\langle\Psi_{n^{\prime}} \mid \Psi_{n}\right\rangle=\delta_{n^{\prime} n}$. The symbol $\{n\}$ indicates that the summation is performed over all possible configuration of fields with $n$ interactions.

The number of particles in the state $\left|\Psi_{n}\right\rangle$ is not directly related to $n$, since high order contributions to the $N$ particles states can be important, but certainly $N \leq M(n):=$ $n(\tilde{N}-1)+1$, where $\tilde{N}$ is the number of particles created or annihilated at each interaction. In Yang-Mills field theory, $\tilde{N}=2 .^{2}$

\footnotetext{
${ }^{2}$ Here diagrams with four external lines are not considered, since they give a nonrenormalizable contribution. However, when all diagrams are summed up, the contribution of the contact interaction is null.
}

We can introduce states of well-defined number of effective partons, $\left|\psi_{N}\right\rangle$, so that

$$
\left|\Psi_{n}\right\rangle=\sum_{N}\left\langle\psi_{N} \mid \Psi_{n}\right\rangle\left|\psi_{N}\right\rangle
$$

The states $\left|\psi_{N}\right\rangle$ are autovectors of $H_{o}$ with fixed number of particles, $N$. Of course, $\left\langle\Psi_{n} \mid \psi_{N}\right\rangle=0$ whenever $N>M(n)$ and $\left\langle\Psi_{N^{\prime}} \mid \Psi_{N}\right\rangle=\delta_{N^{\prime} N}$.

Since the number of partons is fixed and they do not interact but by contact interaction, the states $\left|\psi_{N}\right\rangle$ can be understood as the states of an ideal gas of $N$ partons. Therefore,

$$
\left|\psi_{N}\right\rangle=\mathcal{S}\left|\gamma_{1}, m_{1}, p_{1}, \ldots, \gamma_{N}, m_{N}, p_{N}\right\rangle
$$

where $m_{i}$ and $p_{i}$ are the mass and momentum of the $i$ partonic state, and $\gamma_{i}$ represents all relevant quantum numbers necessary to completely characterize the partonic state. $\mathcal{S}$ is the symmetrization operator acting over fermions and bosons. Since the mass of the effective partons varies continuously, $\mathcal{S}$ gives a negligible modification of the single parton states, so mass and momentum of each parton can vary independently, as far as the total energy is conserved.

Notice that the states with $N$ partons can be obtained in several ways, since

$$
|\Psi(t)\rangle=\sum_{n} \sum_{N}\left\langle\Psi_{n} \mid \Psi(t)\right\rangle\left\langle\psi_{N} \mid \Psi_{n}\right\rangle\left|\psi_{N}\right\rangle,
$$

with $\left\langle\psi_{N} \mid \Psi_{n}\right\rangle \neq 0$ for $M(n) \geq N$. For sufficiently high number of interactions, $n$, there are so many ways to obtain a $N$ particle system that the possibility to get a particular configuration becomes insensitive to the initial state. This situation is similar to that of complex systems where a statistical approach is possible. Therefore, we assume that the probability to get a particular macrostate configuration where the total energy of the system is $E$ and one of the partons has quantum numbers, effective mass, and fourmomentum given by $\left(\gamma_{o}, m_{o}, p_{o}\right)$ can be calculated, aside the statistical weight corresponding to Boltzmann factor, on the sole basis of the number of microstate configurations corresponding to that macrostate, and assumed that all microstates configurations have the same probability to be observed.

The probability to find a state where at least one parton has mass between $m_{o}$ and $m_{o}+d m_{o}$, and momentum coordinates between $p_{o i}$ and $p_{o i}+d p_{o i}$ is given by matrix elements like

$$
\begin{aligned}
& \left\langle\gamma_{o}, m_{o}, p_{o}, \ldots \mid \Psi(t)\right\rangle \\
& \quad=\sum_{n} \sum_{N}\left\langle\Psi_{n} \mid \Psi(t)\right\rangle\left\langle\psi_{N} \mid \Psi_{n}\right\rangle\left\langle\gamma_{o}, m_{o}, p_{o}, \ldots \mid \psi_{N}\right\rangle .
\end{aligned}
$$


We will analyze each of the three brackets in the right-hand side of the equation above individually.

The bracket $\left\langle\Psi_{n} \mid \Psi\right\rangle$ is related to the probability that an effective parton with energy between $E$ and $E+d E$ at time $t=0$ will evolve in such a way that at time $t$ it will generate an arbitrary number of secondary effective partons in a process with $n$ interactions. This probability depends on the probability to find the initial particle with energy between $E$ and $E+d E$, which we write as $P(E) d E$, where $P(E)$ is to be determined, and on the probability that exactly $n$ interaction will occur in the elapsed time, which we write as $G^{n}$. Therefore, we have

$$
\left\langle\Psi_{n} \mid \Psi\right\rangle=G^{n} P(E) d E .
$$

The second bracket depends on the relative number of possibilities to get the configuration with $N$ particles after $n$ interactions, so

$$
\left\langle\psi_{N} \mid \Psi_{n}\right\rangle=C_{N}(n)
$$

with

$$
\sum_{n} C_{N}(n)=1
$$

The last bracket in the expression above is calculated statistically; see the Appendix for technical details. The result is

$f\left(p_{j}\right) d^{4} p_{j}=\frac{1}{8 \pi} \frac{\Gamma(4 N)}{\Gamma(4(N-1))} E^{-4}\left(1-\frac{p_{j}^{0}}{E}\right)^{4 N-5} d^{4} p_{j}$.

The component $p_{j}^{0}$ is the energy of the particle inside the system with $N$ particles, $\varepsilon_{j}$; therefore, $p_{j}^{0}=\varepsilon_{j}$. Then, Eq. (17) can be written as

$$
f\left(\varepsilon_{j}\right) d^{4} p_{j}=A(N) P_{N}\left(\frac{\varepsilon_{j}}{E}\right) d^{4}\left(\frac{p_{j}}{E}\right),
$$

with

$$
P_{N}\left(\frac{\varepsilon_{j}}{E}\right)=\left(1-\frac{\varepsilon_{j}}{E}\right)^{4 N-5}
$$

and

$$
A(N)=\frac{\Gamma(4 N)}{8 \pi \Gamma(4(N-1))} .
$$

From the expression for $A(N)$, we see that the number of states increases with $N^{4}$, so those configurations with large number of particles are favored. The maximum number possible is

$$
M(n)=n(\tilde{N}-1)+1,
$$

so the probability to get a configuration with $N$ particles will be, for sufficiently large $n$, approximately $(N /(n \tilde{N}-n))^{4}$, so using Eqs. (14) and (15), we obtain

$$
\begin{aligned}
\tilde{P}\left(\varepsilon_{j}\right) d^{4} p_{o} d E= & \sum_{n} \sum_{N} G^{n}\left(\frac{N}{n \tilde{N}-n}\right)^{4}\left(1-\frac{\varepsilon_{j}}{E}\right)^{4 N-5} \\
& \times d^{4}\left(\frac{p}{E}\right) P(E) d E
\end{aligned}
$$

Observe that when $N$ is sufficiently large and $\varepsilon_{j} / E$ sufficiently smaller than unit, we have

$\left[1-\frac{\varepsilon_{j}}{E}\right]^{(4 N-5)}\left[1+\frac{\varepsilon_{j}}{E}\right]^{(4 N-5)}=\left[1-\frac{\varepsilon^{2}}{E^{2}}\right]^{(4 N-5)} \sim 1$.

Therefore, we can set

$$
\left[1-\frac{\varepsilon_{j}}{E}\right]^{(4 N-5)}=\left[1+\frac{\varepsilon_{j}}{E}\right]^{-(4 N-5)}
$$

and finally obtain

$$
\begin{aligned}
\tilde{P}(\varepsilon) d^{4} p_{o} d E= & \sum_{n} \sum_{N} G^{n}\left(\frac{N}{n \tilde{N}-n}\right)^{4}\left(1+\frac{\varepsilon}{E}\right)^{-(4 N-5)} \\
& \times d^{4}\left(\frac{p}{E}\right) P(E) d E
\end{aligned}
$$

Below we will show that the general form for the probability densities $\tilde{P}\left(\varepsilon_{j}\right)$ and $P(E)$ can be obtained from considerations about self-similarity.

\section{SELF-SIMILARITY AND FRACTAL STRUCTURE}

The result obtained in Eq. (25) shows that for an ideal gas with finite number of particles, the probability depends on a power-law function of the ratio $\varepsilon_{j} / E$. While this is valid for an ideal gas, where particles have no internal structure, the same result cannot be directly applied to the gas of effective partons because effective partons always have internal structure related to self-energy contributions coming from interaction with the vacuum. We will now investigate how the scaling properties determined by the renormalization group equation can be used to obtain the result for effective partons.

The scaling properties represented by the CallanSymanzik equation demands that effective partons at any scale are similar to any other parton after the proper rescaling is performed. In other words, if partonic properties are expressed in terms of scale free variables they must be described by the same functions of those variables. The energy distribution of a parton, as given by Eq. (25), 
depends on the ratio $\chi=\varepsilon_{j} / E$. Now, let us consider that the system with energy $E$ in which the parton with energy $\varepsilon_{j}$ is one among $N$ constituents, is itself a parton inside a larger system with energy $\mathcal{M}$. Then the ratio $E / \mathcal{M}$ is represented by the same variable, $\chi$, that describes the ration $\varepsilon_{j} / E$. In addition, we can write $P(E)$ in Eq. (25) as $P(E / \mathcal{M})$, and this probability density must follow that same function as $P\left(\varepsilon_{j} / E\right)$, i.e.,

$$
P\left(\frac{\varepsilon_{j}}{E}\right) \sim P\left(\frac{E}{\mathcal{M}}\right) \sim P(\chi),
$$

where the sign $\sim$ indicates that the three functions above are indeed the same function of the scale free variable $\chi$. The same conclusion is valid for any parton with energy $\varepsilon$ which is a component of another parton with energy $\Lambda$, and to let it clear in the following calculations we write $\chi=\varepsilon / \Lambda$. The reasoning just used here is the same that applies to fractal structures, so what we are doing is to introduce the mathematical tools common to fractals studies in the analysis of Yang-Mills fields.

The self-similarity among the partons implies that the probability that the parent parton with mass $E$ inside a larger system with mass $\mathcal{M}$ is similar to the probability given in Eq. (25), so the dependence on $N$ that appears in the exponent must be changed to a parameter $\alpha$, which remains to be determined. This parameter represents the total number of degrees of freedom of the fractal, playing the same role of the exponent $4 N-5$ in the case of the ideal gas. We can write

$$
P\left(\frac{E}{\mathcal{M}}\right)=\left(1+\frac{E}{\mathcal{M}}\right)^{-\alpha}
$$

and the function being integrated in Eq. (25) contains the term

$$
\begin{aligned}
& {\left[P\left(\frac{E}{\mathcal{M}}\right)\right]^{\nu} P_{N}\left(\frac{\varepsilon}{E}\right) d\left(\frac{\varepsilon}{E}\right)} \\
& \quad \sim\left[1+\frac{\varepsilon}{\Lambda}\right]^{-\alpha \nu}\left[1+\frac{\varepsilon}{\Lambda}\right]^{-(4 N-5)} d\left(\frac{\varepsilon}{\Lambda}\right) .
\end{aligned}
$$

Notice that the parameters $\alpha$ and $\nu$ describe the complexity of the interaction involved in the gauge field interaction, and in this sense they measure the sensibility of the effective parton to its internal degrees of freedom.

The self-similarity implies, from relation (28), that

$$
(4 N-5)+\alpha \nu=\alpha,
$$

since the same probability governs any parton distribution. Therefore,

$$
\alpha=\frac{4 N-5}{1-\nu} .
$$

The parameter $\nu$ represents the fraction of total number of degrees of freedom of the state $\left|\psi_{N}\right\rangle$ that is involved in each interaction. Observe that we expect $\nu \leq 1$; therefore, $\alpha$ is positive.

Observe that $q$ is related to the resolution parameter $\nu$ and to the number of particles, $N$. But these two parameters are not independent, since $N$ increases as the resolution increases. In fact, we can write

$$
\Lambda=\alpha \lambda,
$$

where $\lambda$ is a reduced scale, independent of the number of degrees of freedom relevant to the system. Since variable $\chi$ must follow a universal distribution for any parton that is independent of the level in the fractal structure, and since the smallest parton is the one obtained in one-loop approximation, where $N=\tilde{N}$, and therefore is constant, then also $1 / \alpha=\lambda / \Lambda$ is independent of the position the system occupies in the fractal structure, and so $\alpha$ is constant. We can introduce a parameter $q$ such that

$$
\frac{1}{\alpha}=q-1,
$$

with $q>1$ been constant for any parton in the fractal system. Then, we obtain

$$
P(\varepsilon / \lambda)=\left[1+(q-1) \frac{\varepsilon}{\lambda}\right]^{\frac{-1}{q-1}} .
$$

This result shows that the distribution of parton energy created by a system governed by Yang-Mills fields depends only on the ratio between the parton energy, $\varepsilon$, and the energy scale per degree of freedom $\lambda$. Furthermore, it shows that the energy distribution follows the $q$-exponential function commonly found in Tsallis nonextensive statistics.

Similar results have been obtained through a different approach using the concept of thermofractals, introduced in Ref. [17] and studied in details in Ref. [18]. There it is shown that the fractal structure leads to the nonextensive statistics, and it is discussed the relations between thermofractals and Hagedorn's self-consistent thermodynamics developed to study high energy collisions [19,20], and that was extended to nonextensive statistics [21].

In Refs. [16,18], it is discussed that the probability density given by Eq. (33) describes how the energy received by the initial parton flows to its internal degrees of freedom. In the context of the theory developed here, this probability density describes how the energy flows from the initial parton to partons at higher perturbative orders. Since new orders are associated to new vertices, we assume that the $q$ exponential plays the role of an effective coupling constant in the vertex function, i.e.,

$$
\Gamma=\left\langle\Psi_{n+1}\left|g e^{i H_{o} t_{n+1}}\right| \Psi_{n}\right\rangle
$$


with

$$
g=\prod_{i=1}^{\tilde{N}} G\left[1+(q-1) \frac{\varepsilon_{i}}{\lambda}\right]^{-1 /(q-1)}
$$

\section{DISCUSSION AND APPLICATIONS}

As an application of the formalism proposed here, let us consider a vertex in two different orders, as depicted in Fig. 2. The vertex function is

$$
\Gamma_{o}=\left\langle\gamma_{2} p_{2} \gamma_{3} p_{3}\left|g\left(\lambda_{o}\right) e^{i H_{o} t}\right| \gamma_{1} p_{1}\right\rangle .
$$

The next order in perturbative approximation is given by the vertex with one additional loop, which results in a vertex function,

$$
\begin{aligned}
\Gamma= & \left\langle\gamma_{2} p_{2} \gamma_{3} p_{3}\left|g\left(\lambda_{o}\right) e^{i H_{o} t_{3}}\right| \gamma_{2} p_{6}, \gamma_{3} p_{3}, \gamma_{4} p_{4}\right\rangle \\
& \times\left\langle\gamma_{2} p_{6}, \gamma_{3} p_{3}, \gamma_{4} p_{4}\left|g(\lambda) e^{i H_{o} t_{2}}\right| \gamma_{1} p_{5}, \gamma_{4} p_{4}\right\rangle \\
& \times\left\langle\gamma_{1} p_{5}, \gamma_{4} p_{4}\left|g\left(\lambda_{o}\right) e^{i H_{o} t_{1}}\right| \gamma_{1} p_{1}\right\rangle,
\end{aligned}
$$

where the loop involves particles with the same quantum numbers as the initial vertex, as represented by the quantum numbers $\gamma_{1}, \gamma_{2}$, and $\gamma_{3}$, which are repeated in the internal vertex. This expression can be simplified to

$$
\Gamma=\left\langle\gamma_{2} p_{2} \gamma_{3} p_{3}\left|\bar{g} e^{i H_{o} t}\right| \gamma_{1} p_{1}\right\rangle,
$$

where $\bar{g}$ is the effective coupling given by

$$
\bar{g}=g\left(\lambda_{o}\right) e^{i H_{o} t_{3}}\left|\gamma_{2} p_{6}, \gamma_{3} p_{3}, \gamma_{4} p_{4}\right\rangle \Gamma_{M}\left\langle\gamma_{1} p_{5}, \gamma_{4} p_{4}\right| g\left(\lambda_{o}\right),
$$

with

$$
\Gamma_{M}=\left\langle\gamma_{2} p_{6}, \gamma_{3} p_{3}, \gamma_{4} p_{4}\left|g(\lambda) e^{i H_{o} t_{2}}\right| \gamma_{1} p_{5}, \gamma_{4} p_{4}\right\rangle .
$$

Notice that the vertex function in Eq. (38) is similar to the vertex function in Eq. (36), but in the first one we have an effective coupling encompassing the effects of the added loop, as described in Eq. (39). Moreover, the vertex function in Eq. (40) involves particles with the same quantum numbers, $\gamma_{1}, \gamma_{2}, \gamma_{3}$, as in the initial vertex, but

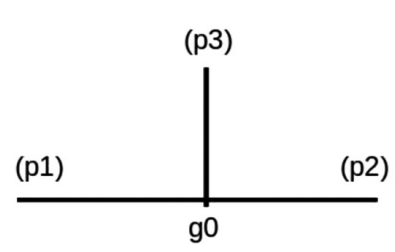

(a) (p3)

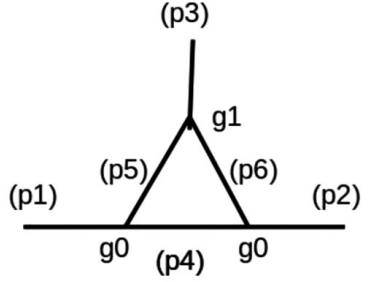

(b)
FIG. 2. Vertex functions at scale (a) $\lambda_{o}$ and (b) $\lambda$. includes the fields of particle 4, which appears in the initial and remains unchanged in the final state, and therefore is not involved in the interaction. The scaling properties of Yang-Mills fields prescribe that apart form multiplying constants related to the fields of particle 4, the vertex function in Eq. (40) is related to the vertex function in Eq. (36) by simple scale rules.

Observe that the effect of the effective coupling as defined by Eq. (35) is to increase the contribution of low energy partons and decrease the contribution of high energy partons. At each vertex, the creation of a parton with energy much higher than that expected for partons at the fractal level, as determined by the scale $\lambda$, is strongly suppressed. The same is valid for the parton annihilation: partons with high energy are more likely to remain in the system with small probability to interact with the less energetic and therefore less massive partons in the medium. Depending on its color content, the heavy parton can reach the surface of the medium and escape. In this way, a complete description of the behavior of those heavy partons must include the possibility of color exchange and coalescence, and some works in this direction, using power-law distributions, have already been done [26]. Keeping these aspects in mind, we continue here with a purely cinematic description of the partonic evolution of QCD. Due to energy-momentum conservation constraint, $\varepsilon_{5}+\varepsilon_{6} \sim \lambda$, so the coupling strongly favors configurations where $\varepsilon_{5} \sim \varepsilon_{6} \sim \lambda / 2$. In this way, the effective coupling controls how the energy transferred to an effective parton is distributed among its internal degrees of freedom.

Note that $\Gamma_{M}$ and $\Gamma_{o}$ are similar, differing only by the presence of the noninteracting field $\left(\gamma_{4} p_{4}\right)$ and by the different energies of the fields at each vertex. The scaling properties of Yang-Mills fields allow us to relate $\Gamma_{M}$ to $\Gamma_{o}$ by an appropriate scale, $\lambda$. This scale must take into account that this is a one-loop contribution, so $\lambda / \lambda_{o}=\tilde{N}^{-1}$, and the coupling constant and fields in $\Gamma_{M}$ must be modified accordingly to $[2,3]$

$$
\phi_{i}(p, \bar{m})=Z_{i}^{-1} \phi_{i}(p, m)
$$

where $m$ is the mass of the parton at scale $\lambda_{o}$ and $\bar{m}$ is its mass at scale $\lambda$. Here $\phi_{i}$ refers to the field of parton $i$, and $Z_{i}$ are multiplicative factors arising from the vertex renormalization.

The renormalization properties impose that $[2,3]$

$$
Z_{i}^{-1}=1+\int_{\lambda_{o}}^{\lambda} \gamma_{i}(m / \lambda, \bar{g}) \frac{d \lambda}{\lambda} .
$$

For one-loop approximation, we have $\lambda=\lambda_{o}+d \lambda$, where $d \lambda<0$, and the integration above turns into

$$
Z_{i}^{-1}=1+\frac{g^{2}}{16 \pi^{2}} \gamma_{i} \log \left(\lambda / \lambda_{o}\right)
$$


from where it follows that

$$
\lambda \frac{\partial \phi_{i}}{\partial \lambda}=\gamma_{i} \phi_{i}
$$

The field $\left(\gamma_{4} p_{4}\right)$ in $\Gamma_{M}$ does not interact at this vertex and it does not need to be scaled, so it will be omitted in the following calculation for the sake of clarity.

The scaling behavior of the $\Gamma(M)$ function is obtained from dimensional analysis, and it results to be $\Gamma_{M}(\lambda)=$ $\left(\lambda / \lambda_{o}\right)^{4}$, after energy-momentum conservation is taken into account, so

$$
M \frac{\partial \Gamma}{\partial \lambda}=d \Gamma
$$

with $d=4$ in the present case. From these considerations and from Callan-Symanzik equation, it results that

$$
\beta_{\bar{g}} \frac{\partial \Gamma}{\partial g}=-\left(d+\gamma_{5}+\gamma_{6}\right) \Gamma .
$$

The contribution to the scaling transformation from $\bar{g}$ appears through $g(\lambda)$, while the other two vertex are at the initial scale, i.e., $g=g\left(\lambda_{o}\right)$. Also, in order to compare with QCD results, we study the behavior of $g(\lambda)$ at $\lambda=\lambda_{o} / \mu$. With the introduction of the scaling factor, $\mu$, the asymptotic limit $\mu \rightarrow \infty$ corresponds to the QCD limit $p \rightarrow \infty$ where asymptotic freedom is expected. From Eq. (35), we have

$$
g(\mu)=\prod_{i=5}^{6} G\left[1+(q-1) \frac{\varepsilon_{i} \mu}{\lambda_{o}}\right]^{-1 /(q-1)} .
$$

Substituting Eq. (47) into Eqs. (40) and (39), we can calculate the beta function in the one-loop approximation taking into account that the derivatives are calculated at $\lambda=\lambda_{o}$ and the asymptotic limit

$$
(q-1) \mu \gg \frac{\lambda_{o}}{\varepsilon_{i}}
$$

is assumed [23]. Then, we obtain

$$
\beta_{\bar{g}}=-\frac{1}{16 \pi^{2}} \frac{1}{q-1} \bar{g}^{3} .
$$

The behavior of $\beta_{\bar{g}}$ as a function of $g$ and as a function of the scale $\mu$ is displayed in Fig. 3, alongside the behavior of $g$ versus $\mu$.

Scaling properties of QCD have been extensively studied in the one-loop approximation. The beta function for $\mathrm{QCD}$ is $[22,23]$

$$
\beta_{\mathrm{QCD}}=-\frac{\bar{g}^{3}}{16 \pi^{2}}\left[\frac{11}{3} c_{1}-\frac{4}{3} c_{2}\right],
$$

where

$$
c_{1} \delta_{a b}=f_{a c d} f_{b c d}
$$

and

$$
c_{2} \delta_{a} b=\operatorname{Tr}\left(T_{a} T_{b}\right),
$$

therefore relating the entropic index, $q$, from Tsallis statistics to fundamental parameters of the field theory.

Quantitatively, the parameters $c_{1}$ and $c_{2}$ are related to the number of colors and flavors by $c_{1}=N_{c}$ and $c_{2}=N_{f} / 2$. Using $N_{c}=N_{f} / 2=3$, we get

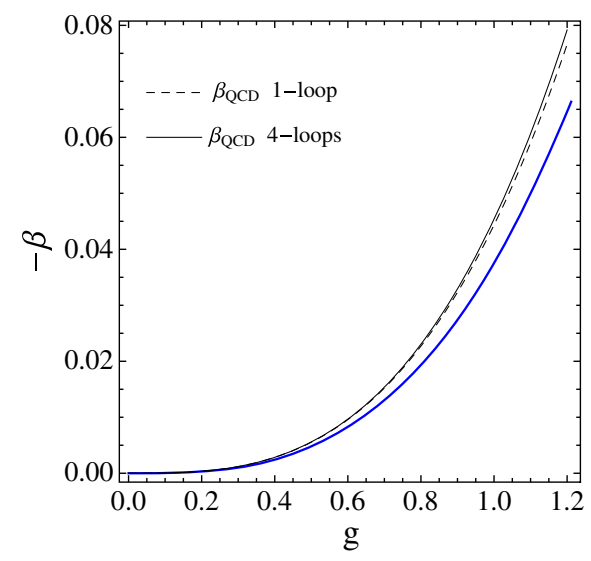

(a)

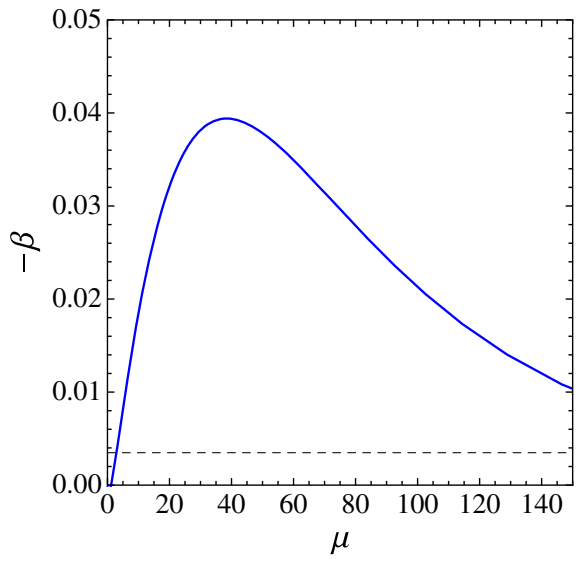

(b)

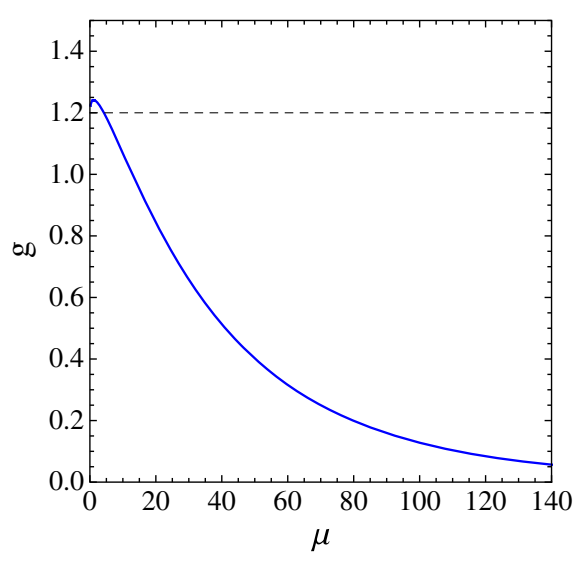

(c)

FIG. 3. Behavior of (a) beta function against effective coupling, as given by Eq. (49), (b) the logarithmic derivative of the effective coupling with respect to the scale $\mu$, obtained by the same equation when the dependence of $g$ on $\mu$ is considered, and (c) effective coupling, as calculated by Eq. (47). The values $G=752$ and $\varepsilon_{5}=\varepsilon_{6}=\lambda / 2$ in (a) and $G=3.67$ were used in the computation of plots (b) and (c). The dashed line represents the value from QCD in one-loop approximation [22,23], and the dash-dotted line represents $\beta$ as calculated in four-loop approximation [24,25]. The different value for the constant $G$ in plot (a) corresponds to a change of scale in the $g$ axis and is chosen to allow a better visualization of the behavior of the curves. 


$$
\frac{11}{3} c_{1}-\frac{4}{3} c_{2}=7
$$

which leads to $q=1.14$. From experimental data analysis [27-29], we have $q=1.14 \pm 0.01$, showing a good agreement between theory and experiments.

\section{THE BETA FUNCTION AND THE EFFECTIVE COUPLING}

The discussion made so far refers to an idealized fractal structure where the mass of the field theory is null or negligible. Let us now discuss the effects of finite parton mass in the theoretical approach used here and what are the limits of its applicability.

The scale $\lambda$ in the effective coupling expression is a parameter that indicates the resolution adopted for studying the fractal structure: when fluctuations of internal energy are small compared to $\lambda$, they have small or negligible effects on the system. A consequence of the physical meaning of this parameter is that, when there is a finite mass, $m$, as the current quark mass in QCD, it is meaningless to adopt $\lambda<m$. Then, there is a critical value, $\mu_{c}$ for the scaling factor, such that

$$
\mu_{c}=\frac{\lambda_{o}}{m} .
$$

Since $\lambda_{o}$ in QCD is usually adopted of the order of the pion mass, which is much larger than the current quark mass, it means that $\mu_{c}$ is very large and we are in the region of the asymptotic freedom; therefore, the effects of the finite quark mass are negligible. In the cases where $\mu_{c}$ is not large enough, we can redefine the scale factor as

$$
\mu=\frac{\lambda_{o}}{\lambda-\lambda_{c}},
$$

with $\lambda_{o}, \lambda>\lambda_{c}$.

The modifications to the scale $\lambda$ and scaling factor $\mu$ mentioned above allow us to extend our approach to a region where the fractal structure is not valid anymore and has to be considered as a phenomenological approach to the more fundamental problem of a structural transition, which may be related to a nonthermal phase transition [30], but it is important to notice that for the case of hot systems, which is our focus here, the effects of quark finite mass are negligible. This phase transition might be associated to the trace anomaly, and in this context it is interesting to notice that the nonextensive generalization of Hagedorn's self-consistent theory is able to describe trace anomaly and results in good agreement with LQCD calculations [31].

It is interesting to note that the scaling invariance can be used in two ways: one can fix a value for $\lambda$, based on experimental resolution or some physical change in the system that makes the scale invariance invalid or useless, and then the several fractal layers are obtained by considering systems of large and large energies corresponding to different values of $\Lambda$; the other way is to fix the total energy and let $\lambda$ vary, meaning that the system is observed with better resolution, and therefore deeper layers of the system are investigated, i.e., the better the resolution, the lower the $\lambda$. The first way finds application in high energy collisions, and the second, in studies of hadron structure.

The results obtained here have shown that a system with fractal structure, similar to the thermofractals introduced in Ref. [17] and studied in [18], can be understood as a natural consequence of the renormalization group invariance of gauge field theories. There is shown that the fractal structure leads to the nonextensive statistics, and it is discussed the relations between thermofractals and Hagedorn's selfconsistent thermodynamics developed to study high energy collisions $[19,20]$, and that was extended to nonextensive statistics [21]. This fractal structure has been already used to investigate properties of hadrons [32], phase transition in hot hadronic matter [33], and neutron stars [34]. The power-law distribution of energy and momentum, which is a direct consequence of the fractal structure, was used to describe $p_{T}$ distributions from high energy collisions experiments $[28,29,35]$ and to describe hadron mass spectrum [28,36]. The results obtained here give a stronger basis for the interpretation of those experimental and phenomenological studies in terms of nonextensive statistics.

\section{MULTIPLICITY}

It is possible to understand, from the considerations made here, that the fractal structure of YMF is the basis for investigations of hadron properties [32], phase-transition in hot hadronic matter [33], neutron stars [34], and cosmicray [37]. These phenomenological approaches are, in fact, implementations of scaling symmetries observed in Yang-Mills fields. The fractal structure also allows the understanding of the self-similarity [38-40] and scaling properties observed in high energy experimental data. In fact, these findings are direct consequences of the scaling properties of YMF, as discussed here. Moreover, the fact that the entropic index, $q$, is obtained from well-known field-theoretical parameters; the results we have obtained allow a new interpretation of Tsallis statistics in terms of fractal structure in the same lines it was obtained in thermofractals approach [36].

The fractal structure presents at least one fractal dimension, and the Haussdorf dimension is a characteristic dimension that can be calculated by using the box-counting technique [7], where the dimension $D$ is related to the number of boxes, $\mathcal{N}$, necessary to completely cover all possible values for the measured quantity and $D_{t}$ is the topological dimension. At some scale $r$, these quantities are related by [7]

$$
\mathcal{N} r^{-D} \propto r^{-D_{t}} .
$$


In our case, $D_{t}=1$ since we are dealing with system energy as a measure. The procedure to obtain the Haussdorf dimension is similar to that followed in Ref. [17]. The average energy of the partons at the scale $\lambda$, already introduced as the energy scale per degree of freedom, is

$$
\langle\varepsilon\rangle=\int_{0}^{\infty} \varepsilon P(\varepsilon) d \varepsilon=\frac{\lambda}{2 q-1} .
$$

The ratio between the average energy of the components and the parent system energy

$$
R=\frac{\langle\varepsilon\rangle}{E}
$$

is related to the level of the fractal structure relative to the scale $\lambda$ by

$$
R^{n}=\frac{\lambda}{E}=r .
$$

The number of boxes with length $\lambda$ necessary to completely cover the possible range of energies in which the fractal components can be found is $\mathcal{N}=\tilde{N}^{n}$. Then, it follows from Eq. (59) that

$$
n=\frac{\log r}{\log R} .
$$

Equation (59) also shows that, in terms of the scales, the energy of the system varies as $E \sim r^{-1}$. Let us now write the dependence of the parton energies at scale $\lambda$ as $\varepsilon \sim r^{-D}$; then

$$
\mathcal{N} r^{-D} \propto r^{-1}
$$

therefore,

$$
D-1=n \frac{\log \tilde{N}}{\log r} .
$$

From Eq. (60), it follows that

$$
D-1=\frac{\log \tilde{N}}{\log R} .
$$

From Eqs. (57) and (58), and using $E=\lambda_{r} /(q-1)$, we get

$$
R=\frac{q-1}{2 q-1}
$$

Using the value $q-1=1.14$, it follows that $D=0.69$. This result is in good agreement with the fractal dimension observed in intermittency analyses of high energy experimental data $[41,42]$. These analyses allow to access fractal dimensions by studying the behavior of cummulants of the measured distributions [43-50], and the systematic analysis show that for $p p$ collisions there is a good agreement between the value obtained from the theory with those resulting from experimental data analyses.

The fractal dimension gives the behavior of the parton energy with the energy scale, $r$, i.e., while the total energy goes as $E \propto r^{-1}$, the partons observed at scale $\lambda$ have energies that depend on the scale as $\varepsilon \propto r^{-D}$. A more direct way to access the fractal dimension is the particle multiplicity. In fact, being $\mathcal{M}$ the particle multiplicity, we have

$$
\mathcal{M}\langle\varepsilon\rangle=E .
$$

From the dimensional behavior obtained above, we get

$$
\mathcal{M}=E^{1-D} .
$$

For the case of hadrons, as we have seen, $q=1.14$ and $D=0.69$, so we obtain $\mathcal{M} \propto E^{0.31}$, which is in excellent agreement with the result obtained for $p p$ collision at high energy [51], which gives, for a power-law fit, an exponent corresponding to $1-D=0.302$.

\section{CONCLUSIONS}

In the present work, the scaling properties of Yang-Mills fields are analyzed under the light of the concept of fractals. It is shown that those scaling properties lead to the formation of a fractal structure, and using a thermodynamical hypothesis similar to that used in LQCD we obtain Tsallis distributions that have been associated to the longtail distributions observed in high energy data. The entropic index, $q$, which in Tsallis statistics is in most cases a parameter to be determined experimentally, in the present case can be determined completely in terms of the fundamental parameters of the field theory.

The result is used to obtain, by a recurrence formula that reflects the self-similar features of the fractal, the effective coupling which is expressed in terms of a scale dependent formula. Applying this expression for the case of QCD in the one-loop approximation, the entropic index is calculated, for the first time, from the numbers of colors and flavors. The result is shown to be in good agreement with the value obtained for $q$ by fitting Tsallis distributions to data. From the analysis of the fractal structure, we obtain the fractal dimension associated to Yang-Mills fields, which is determined completely in terms of the field theory parameters. The fractal dimension is calculated for QCD and the result is in good agreement with the value obtained from intermittency analysis of high energy distributions.

Finally, it is shown that the fractal dimension allows us to determine the behavior of the particle multiplicity against the collision energy, and we obtain a result that is in good agreement with that observed in high energy collisions.

In summary, we have shown in the present work that renormalizable field theories lead to fractal structures, which can be studied, from a thermodynamical point of view, with Tsallis statistics. A recursive method allows to perform nonperturbative calculations to describe the 
particles structure governed by the gauge theory. In the case of multiparticle production, the calculations lead to a thermodynamical description where nonextensive statistics must be used. The results obtained here give a solid basis from QCD to the use of nonextensive self-consistent thermodynamics to describe properties of strong interacting systems and to the use of thermofractal structure to describe hadrons.

\section{ACKNOWLEDGMENTS}

A. D. would like to thank the University of Granada, where part of this work has been done, for the hospitality and financial support under a grant of the Visiting Scholars Program of the Plan Propio de Investigación of the University of Granada. He also acknowledges the hospitality at Carmen de la Victoria. A. D. and D. P. M. are partially supported by the Conselho Nacional de Desenvolvimento Cientfico e Tecnolgico (CNPq-Brazil) and by Project INCTFNA Proc. No. 464898/2014-5. A. D. is partially supported by FAPESP under Grant No. 2016/17612-7. The work of E. M. is supported by the Spanish MINEICO and European FEDER funds under Grants No. FIS2014-59386-P and No. FIS2017-85053-C2-1-P, by the Junta de Andalucía under Grant No. FQM-225, and by the Consejería de Conocimiento, Investigación y Universidad of the Junta de Andalucía and European Regional Development Fund Grant No. SOMM17/6105/UGR. The research of E. M. is also supported by the Ramón y Cajal Program of the Spanish MINEICO under Grant No. RYC-2016-20678.

\section{APPENDIX: CALCULATION OF DENSITY OF STATES}

Given a system with $N$ free particles with Hamiltonian,

$$
H=\sum_{i=1}^{N} p_{i}^{0},
$$

where $p_{i}^{\mu}=\left(p_{i}^{0}, \vec{p}_{i}\right)$ is the fourth momentum of particle $i$, the goal is to compute the density of states $\rho(p)$, with the normalization condition

$$
1=\int d^{4 N} p \rho(p) .
$$

In the following, we will not assume a fixed value for the mass $m_{i}$ of particle $i$, where $m_{i}^{2}=p^{\mu} p_{\mu}$, so that $p_{i}^{0}$ and $\vec{p}_{i}$ are variables that may change independently each other. Let us compute the phase space volume of region $H \leq E$, i.e.,

$$
\Omega_{N}(E)=\int d^{4 N} p \Theta\left(E-\sum_{i=1}^{N} p_{i}^{0}\right),
$$

where $\Theta(x)$ is the step function. We can express the integral in the form
$\Omega_{N}(E)=\int d^{N} p^{0} \Theta\left(E-\sum_{i=1}^{N} p_{i}^{0}\right) \prod_{i=1}^{N} \int d^{3} p_{i} \Theta\left(p_{i}^{0}-\left|\vec{p}_{i}\right|\right)$.

Note that for particle $i$ one has $\vec{p}_{i}^{2}=\left(p_{i}^{0}\right)^{2}-m_{i}^{2}$. Because $m_{i}$ is not fixed to a particular value, the limit of the integration in the $d^{3} p_{i}$ integral is $0 \leq\left|\vec{p}_{i}\right| \leq p_{i}^{0}$, and this has been expressed with the corresponding step function in Eq. (A4). This integral can be easily computed to give $\int d^{3} p_{i} \Theta\left(p_{i}^{0}-\left|\vec{p}_{i}\right|\right)=\frac{4}{3} \pi\left(p_{i}^{0}\right)^{3}$, so that Eq. (A4) turns out to be

$\Omega_{N}(E)=\left(\frac{4 \pi}{3}\right)^{N} \int d^{N} p^{0}\left(\prod_{i=1}^{N}\left(p_{i}^{0}\right)^{3}\right) \Theta\left(E-\sum_{i=1}^{N} p_{i}^{0}\right)$.

From dimensional analysis, one can see that the result of this integral should be of the form

$$
\Omega_{N}(E)=c_{N} \pi^{N} E^{4 N},
$$

where $c_{N}$ are some coefficients to be determined, and the factor $\pi^{N}$ has been explicitly extracted for convenience. It is possible to obtain the coefficients $c_{N}$ by mathematical induction. In the case $N=1$, one can easily check that

$$
\Omega_{1}(E)=\frac{1}{3} \pi E^{4},
$$

so that $c_{1}=1 / 3$. Assuming that the expression of $\Omega_{N}(E)$ is known, let us compute $\Omega_{N+1}(E)$. It writes

$$
\begin{aligned}
\Omega_{N+1}(E) & =\int d^{4} p_{N+1} \int d^{4 N} p \Theta\left(E-\sum_{i=1}^{N+1} p_{i}^{0}\right) \\
& =\int d^{4} p_{N+1} \int d^{4 N} p \Theta\left(E-p_{N+1}^{0}-\sum_{i=1}^{N} p_{i}^{0}\right) \\
& =\int d^{4} p_{N+1} \Omega_{N}\left(E-p_{N+1}^{0}\right),
\end{aligned}
$$

where in the last equality we have performed the integral only in the momentum of particles $i=1, \ldots, N$. The integral in $d^{4} p_{N+1}$ can be easily performed following similar steps as above, i.e.,

$$
\begin{aligned}
\Omega_{N+1}(E)= & \int d^{4} p_{N+1} \Omega_{N}\left(E-p_{N+1}^{0}\right) \\
= & \int_{0}^{E} d p_{N+1}^{0} \Omega_{N}\left(E-p_{N+1}^{0}\right) \\
& \times \int d^{3} p_{N+1} \Theta\left(p_{N+1}^{0}-\left|\vec{p}_{N+1}\right|\right) \\
= & \frac{4 \pi}{3} \int_{0}^{E} d p_{N+1}^{0}\left(p_{N+1}^{0}\right)^{3} \Omega_{N}\left(E-p_{N+1}^{0}\right) .
\end{aligned}
$$


Using now Eq. (A6), this integral can be easily performed, and the result is

$$
\Omega_{N+1}(E)=8 \pi^{N+1} \frac{\Gamma(4 N+1)}{\Gamma(4 N+5)} c_{N} E^{4(N+1)} .
$$

This result should be identified with $\Omega_{N+1}(E)=c_{N+1} \pi^{N+1} \times$ $E^{4(N+1)}$, and from it one obtains a relation between $c_{N+1}$ and $c_{N}$, which can be used iteratively to obtain

$$
c_{N}=\frac{4 ! \cdot 8^{N-1}}{(4 N) !} c_{1}=\frac{8^{N}}{(4 N) !} .
$$

In the last equality, we have used that $c_{1}=1 / 3$. Finally, the result of Eq. (A3) is

$$
\Omega_{N}(E)=\frac{(8 \pi)^{N}}{(4 N) !} E^{4 N} .
$$

Let us introduce a constant distribution in the microcanonical ensemble,

$$
\rho(p)= \begin{cases}C, & \text { for } E \leq H \leq E+\Delta E \\ 0 & \text { otherwise }\end{cases}
$$

that should be normalized to 1 as indicated in Eq. (A2). Then, one finds that

$$
1=C \cdot\left[\Omega_{N}(E+\Delta E)-\Omega_{N}(E)\right],
$$

so that

$$
C=\frac{1}{\Omega_{N}(E+\Delta E)-\Omega_{N}(E)} .
$$

The probability distribution for particle $j$ to have momentum $p_{j}^{\mu}$ is obtained by integrating the joint distribution $\rho\left(p_{1}, \ldots, p_{N}\right)$ over all the variables except $p_{j}$. Then, one has

$$
\begin{aligned}
f\left(p_{j}\right) d^{4} p_{j}= & d^{4} p_{j} \int d^{4(N-1)} p \rho(p) \Theta(E \leq H \leq E+\Delta E) \\
= & d^{4} p_{j} \int d^{4(N-1)} p \rho(p) \\
& \times \Theta\left(E-p_{j}^{0} \leq \sum_{i=1}^{N^{\prime}} p_{i}^{0} \leq E+\Delta E-p_{j}^{0}\right), \quad
\end{aligned}
$$

where the prime in $\sum_{i=1}^{N^{\prime}}$ means that the term $i=j$ should be excluded in the summation. Using the result above for $\rho(p)$, this can be expressed in the form $f\left(p_{j}\right) d^{4} p_{j}=d^{4} p_{j} \frac{\Omega_{N-1}\left(E+\Delta E-p_{j}^{0}\right)-\Omega_{N-1}\left(E-p_{j}^{0}\right)}{\Omega_{N}(E+\Delta E)-\Omega_{N}(E)}$.

Finally, by using the explicit expression of $\Omega_{N}(E)$, given by Eq. (A12), and taking the limit $\Delta E \rightarrow 0$, one arrives at the final result

$f\left(p_{j}\right) d^{4} p_{j}=d^{4} p_{j} \frac{1}{8 \pi} \frac{\Gamma(4 N)}{\Gamma(4(N-1))} E^{-4}\left(1-\frac{p_{j}^{0}}{E}\right)^{4 N-5}$.

One can easily check that this result is correctly normalized, i.e., $\int d^{4} p_{j} f\left(p_{j}\right)=1$. The density of states with energy $p_{j}^{0}$ can be computed by integrating in $d^{3} p_{j}$, and the result is

$$
\begin{aligned}
f\left(p_{j}^{0}\right) d p_{j}^{0} & =d p_{j}^{0} \int d^{3} p_{j} f\left(p_{j}\right) \Theta\left(p_{j}^{0}-\left|\vec{p}_{j}\right|\right) \\
& =d p_{j}^{0} \frac{1}{6} \frac{\Gamma(4 N)}{\Gamma(4(N-1))} E^{-1}\left(\frac{p_{j}^{0}}{E}\right)^{3}\left(1-\frac{p_{j}^{0}}{E}\right)^{4 N-5} .
\end{aligned}
$$

If one considers the system in the rest mass frame, then $E=M$, i.e., the total energy of the system is equal to its mass. In addition, $p_{j}^{0}=\varepsilon_{j}$ is the energy of particle $j$. Then, we can express this result in the equivalent form,

$$
f\left(\varepsilon_{j}\right) d \varepsilon_{j}=\frac{1}{6} \frac{\Gamma(4 N)}{\Gamma(4(N-1))}\left(\frac{\varepsilon_{j}}{M}\right)^{3}\left(1-\frac{\varepsilon_{j}}{M}\right)^{4 N-5} d\left(\frac{\varepsilon_{j}}{M}\right) .
$$

Notice that the density of states with one particle with energy $p_{j}^{0}$ can be obtained by integrating on the vector momentum coordinates, resulting in

$$
f\left(p_{j}^{0}\right) d p_{j}^{0}=\frac{1}{6} \frac{\Gamma(4 N)}{\Gamma(4(N-1))} E^{-1}\left(\frac{p_{j}^{0}}{E}\right)^{3}\left(1-\frac{p_{j}^{0}}{E}\right)^{4 N-5} d p_{j}^{0} .
$$

Observe that if we write $E=(4 N-5) \mu$, and take the limits $N \rightarrow \infty$ and $\mu \rightarrow 0$ keeping constant $N \mu=k T$, we obtain the exponential factor usually found in the thermodynamical limit. Here, however, we cannot take such limit because $N<M(n)$. 
[1] M. A. Matwi, Physics (Basel, Switz.) 1, 339 (2019).

[2] F. J. Dyson, Phys. Rev. 75, 1736 (1949).

[3] M. Gell-Mann and F. E. Low, Phys. Rev. 95, 1300 (1954).

[4] J. C. Ward, Proc. Phys. Soc. London Sect. A 64, 54 (1951).

[5] B. B. Mandelbrot, The Fractal Geometry of Nature (WH Freeman, New York, 1983).

[6] G. West, Scale, The Universal Laws of Life, Growth, and Death in Organisms, Cities, and Companies (Penguin Books, New York, 2018).

[7] K. Falconer, Fractals, A Short Introduction (Oxford University Press, Oxford, 2013).

[8] C. Tsallis, J. Stat. Phys. 52, 479 (1988).

[9] M. Gell-Mann and C. Tsallis, Nonextensive Entropy: Interdisciplinary Applications (Oxfor University Press, Oxford, 2004).

[10] H. D. Politzer, Phys. Rep. 14, 129 (1974).

[11] H. Georgi and H. D. Politzer, Phys. Rev. D 9, 416 (1974).

[12] D. Gross and F. Wilczek, Phys. Rev. D 9, 980 (1974).

[13] C. G. Callan, Jr, Phys. Rev. D 2, 1541 (1970).

[14] K. Symanzik, Commun. Math. Phys. 18, 227 (1970).

[15] K. Symanzik, Commun. Math. Phys. 23, 49 (1971).

[16] A. Deppman and E. Megias, Physics 1, 103 (2019).

[17] A. Deppman, Phys. Rev. D 93, 054001 (2016).

[18] A. Deppman, T. Frederico, E. Megías, and D. P. Menezes, Entropy 20, 633 (2018).

[19] R. Hagedorn, CERN Report No. TH. 520 65/166/5, 1965.

[20] R. Hagedorn, Nuovo Cimento Suppl. 3, 147 (1965).

[21] A. Deppman, Physica (Amsterdam) 391A, 6380 (2012).

[22] D. J. Gross and F. Wilczek, Phys. Rev. Lett. 30, 1343 (1973).

[23] H. D. Politzer, Phys. Rep. 14, 129 (1974).

[24] T. van Ritbergen, J. A. M. Vermaseren, and S. A. Larin, Phys. Lett. B 400, 379 (1997).

[25] M. Czakon, Nucl. Phys. B710, 485 (2005).

[26] X. Feal, C. Pajares, and R. A. Vazquez, Phys. Rev. C 99, 015205 (2019).

[27] I. Sena and A. Deppman, Eur. Phys. J. A 49, 17 (2013).

[28] L. Marques, E. Andrade II, and A. Deppman, Phys. Rev. D 87, 114022 (2013).
[29] L. Marques, J. Cleymans, and A. Deppman, Phys. Rev. D 91, 054025 (2015).

[30] R. Peschanski, Nucl. Phys. B327, 144 (1989).

[31] A. Deppman, J. Phys. G 41, 055108 (2014).

[32] P. H. G. Cardoso, T. Nunes da Silva, A. Deppman, and D. P. Menezes, Eur. Phys. J. A 53, 191 (2017).

[33] E. Megías, D. P. Menezes, and A. Deppman, Physica (Amsterdam) 421A, 15 (2015).

[34] D. P. Menezes, A. Deppman, E. Megías, and L. B. Castro, Eur. Phys. J. A 51, 155 (2015).

[35] M. Rybczynski, Z. Wlodarczyk, and G. Wilk, J. Phys. G 39, 095004 (2012).

[36] A. Deppman, Universe 3, 62 (2017).

[37] G. Yalcin and C. Beck, Sci. Rep. 8, 1764 (2018).

[38] M. Tokarev and I. Zborovsky, EPJ Web Conf. 141, 02006 (2017).

[39] I. Zborovsky and M. V. Tokarev, Phys. Rev. D 75, 094008 (2007).

[40] G. Wilk and Z. Włodarczyk, Phys. Lett. B 727, 163 (2013).

[41] A. Bialas and R. Peschanki, Nucl. Phys. B273, 703 (1986).

[42] A. Bialas and R. Peschanki, Nucl. Phys. B308, 857 (1988).

[43] R. C. Hwa, Phys. Rev. D 41, 1456 (1990).

[44] R. C. Hwa and J. Pan, Phys. Rev. D 45, 1476 (1992).

[45] S. Hegyi, Phys. Lett. B 318, 642 (1993).

[46] I. M. Dremin and R. C. Hwa, Phys. Rev. D 49, 5805 (1994).

[47] S. Hegyi and T. Csörgö, Phys. Lett. B 296, 256 (1992).

[48] N. G. Antoniou, N. Davis, and F. K. Diakonos, Phys. Rev. C 93, 014908 (2016).

[49] E. A. De Wolf, I. M. Dremin, and W. Kittel, Phys. Rep. 270, 1 (1996).

[50] W. Kittel and E. A. De Wolf, Soft Multihadron Dynamics (World Scientific, Singapore, 2005).

[51] E. K. G. Sarkisyan, A. N. Mishra, R. Sahoo, and A. S. Sakharov, Phys. Rev. D 93, 054046 (2016).

Correction: A journal source listing was not set as a reference and has now been fixed as Ref. [1], enabling DOI linkage. 